\title{
Schwannoma of the Brachial Plexus Presented as a Neck Mass: A Case Report and Review of the Literature
}

\author{
Aslan Ahmadi, Hengameh Hirbod, Mostafa Cheraghipoor, Farzad Izadi \\ Department of Ear Nose \& Throat, Hazrate Rasool Hospital, Tehran, Iran \\ Email:dr.aslan ahmadi@yahoo.com, hehirbod@yahoo.com, mostafa.cheraghipoor@yahoo.com, \\ izadimd@yahoo.com
}

Received 15 February 2015; accepted 4 March 2015; published 10 March 2015

Copyright $@ 2015$ by authors and Scientific Research Publishing Inc.

This work is licensed under the Creative Commons Attribution International License (CC BY). http://creativecommons.org/licenses/by/4.0/

(c) (i) Open Access

\begin{abstract}
Schwannomas of the head and neck as well as brachial plexus primary tumors are both uncommon entities, and combination of these conditions is quite rare. Schwannomas of the brachial plexus are usually asymptomatic and they present as slowly enlarging masses in the supra- or infraclavicular regions. Although imaging plays a routine role in the detection of these neoplasms, identification of the nerve origin is not often feasible until the time of surgery. Definitive diagnosis is based on histopathological features with presence of spindle-shaped Schwann cells. We report a case of a middle aged woman with left lateral mid-neck mass, which based on the clinical findings, was provisionally diagnosed as a painless lymphadenopathy. Ensuing excisional biopsy revealed the brachial plexus as the origin of a tumor, which subsequently was confirmed to be a schwannoma with microscopic evaluation. The course of disease was complicated with upper brachial plexus injury which was recovered by sural nerve graft.
\end{abstract}

\section{Keywords}

Schwannoma, Cervical Schwannoma, Brachial Plexus Tumors, Extracranial Schwannoma

\section{Introduction}

Schwannomas, also termed as neurilemmomas, are benign nerve sheath neoplasms that originate from peripheral, cranial or autonomic nerves. These are well encapsulated tumors and malignant transformation is extremely rare. Tumors involving head and neck region consist of around $25 \%$ of all cases, usually originated from cranial (V, VII, IV, X, XI and XII) nerves, sympathetic or peripheral nerves [1]-[4]. These lesions are generally presented 
as a cosmetic deformity, an asymptomatic mass or symptoms related to nerve compression [5]. Advanced imaging investigations, such as magnetic resonance imaging (MRI) and/or computed tomography (CT) scan, are particularly useful in diagnosing these neoplasms and have become the routine studies for these patients [6]. Microscopically, pathological features of these tumors are unique, composed of spindle Schwann cells forming hyper- or hypocellular areas (Antoni A and Antoni B, respectively) [1].

Schwannomas of the brachial plexus are rare and due to their rarity, as well as the complex anatomical situation, they may pose diagnostic or surgical challenges [5] [7]. Proper diagnosis of the tumor must be established prior to surgery as it can be mistaken with a lymphadenopathy [8]. Previous reports showed that a majority of cases with schwannoma involving cranial nerves or sympathetic nerve were associated with a neck mass [2] [6] [9]-[11], while cases with brachial plexus tumors commonly presented as supraclavicular, infraclavicular or neck root swelling, with or without neurological manifestation [3]-[5] [7] [8] [12]-[14].

In this study, we report a middle aged woman with brachial plexus schwannoma who was presented as midcervical mass.

\section{Case Report}

A 42-year-old lady presented with a left neck progressively enlarging swelling for three months duration. There was no history of trauma, pain, hoarseness, dysphagia, syncopal attacks or any constitutional symptoms. Systemic physical examination was normal, however locally; there were a $2 \times 2 \mathrm{~cm}$ firm, nonpulsatile and fairly mobile mass located at the left mid-cervical region. It was not tender, warm or fluctuant and clinically resembled an anterior cervical lymphadenopathy. Otoscopic and oropharynx examination as well as indirect laryngoscopy revealed no abnormality or any source of infection. She was neurologically intact with no evidence of sensory loss and myotomal weakness or abnormal reflexes. Blood investigations showed normal values. A computed tomography (CT) of the neck was performed and discovered a well-defined mass in left lateral cervical region measuring $2 \times 2 \mathrm{~cm}$ at the level of fourth to fifth cervical vertebra (Figure 1). A fine-needle aspiration was performed, which was inconclusive.

Patient was planned for an excisional biopsy and using a left transcervical approach, the entire mass was resected. Intraoperatively, a well-circumscribed mass, measuring $1.5 \times 2 \times 2 \mathrm{~cm}$ was identified, with its bundles passing through the fascicles of the brachial plexus.

Postoperatively she sustained weakness of shoulder abduction, elbow flexion and supination and an upper brachial plexus injury was suspected. She underwent the second operation aiming to explore the existing neural defect and a partially injured C5 nerve root and upper trunk of the brachial plexus was detected. The injured nerves were resected and sural nerve graft was carried out using a microsurgical technique.

Pathological assessment revealed encapsulated lesion composed of neoplastic spindle cells with basophilic nuclei (Schwann cells), organized in patternless alternative hyper and hypo-cellular areas, representing Antoni A and Antoni B patterns, respectively (Figure 2). There were no mitotic figures or necrosis. The final diagnosis
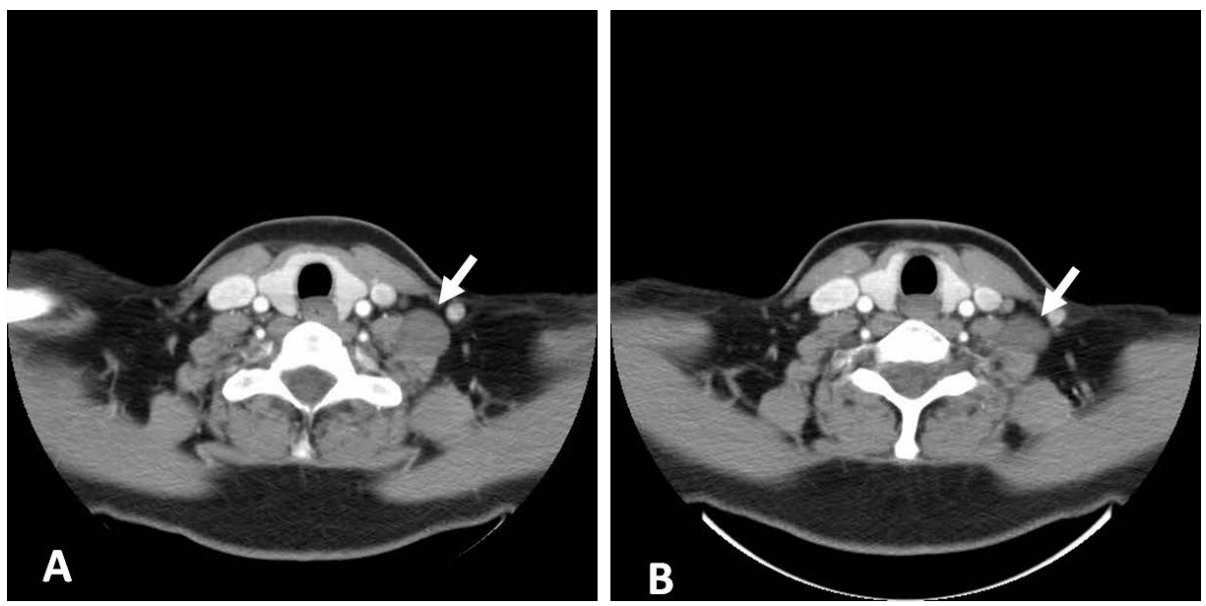

Figure 1. Contrast enhanced computed tomography (CT) scan (axial view) showing the isodense lesion in left cervical region with minimal enhancement (white arrows). 


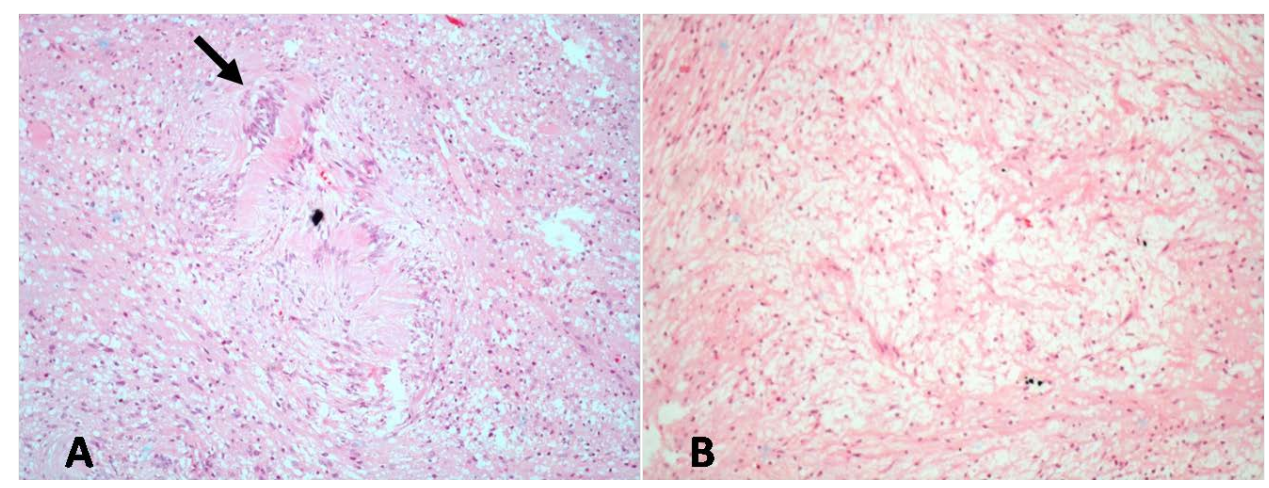

Figure 2. Microscopic examination showed a spindle cell neoplasm with the composition of a hypercellular area, Antoni A (A) and a hypocellular area, Antoni B (B) (H \& E stain, $\times 400)$. Note the palisading nuclei of the spindle cells surrounding pink areas, known as verocay bodies, representing Antoni A pattern (black arrow).

was schwannoma.

At 4 months follow up after discharge, she was symptom free, without any residual neurological deficit.

\section{Discussion}

Primary tumors arising in the brachial plexus are rare [15]. These neoplasms may manifest as pain in the shoulder or upper limb, sensory/motor disturbances or an asymptomatic swelling. Among these primary tumors, schwannoma and neurofibromatosis are the two most common neoplasms and both of which are benign and eccentrically arise from neural sheath [5] [15] [16]. Although schwannomas typically present as a solitary tumor, neurofibromas usually occur in the context of neurofibromatosis type I [5].

Schwannomas are usually indolent neoplasms that originate from cranial, peripheral or autonomic nerve sheaths. The eccentric position of the tumor in the nerve is probably contributing to the reason why the majority of cases with schwannoma, as in our case, are neurologically spared. However, these tumors may have been investigated for space occupying lesion or nerve compression, months or even years prior to final diagnosis [3] [4]. Even more complicated presentations, such as a cystic pectoral lesion [12] or axillary [7] masses have also been reported. Considering the complexity of the regional anatomy, surgical resection of this tumor is a technically challenging procedure that has the potential for devastating neurological complications. In order to achieve the best result, an accurate preoperative planning, using imaging techniques such as MRI or CT scan is highly recommended.

We could not find any published evidence regarding the precise incidence of postoperative neurological complications; however, the review of the reported cases shows that temporary sensory or motor deficit frequently occurs after surgical resection of the schwannoma [3] [5]-[7]. There are scattered reports of long term or permanent motor impairment after this operation as well [5]. Our patient developed a neurological deficit postoperatively that required us to perform the surgical exploration to find out underlying reason, which was discontinuation of the C5 nerve root and upper trunk of the plexus. Neurological and functional outcome of this patient was evaluated as excellent, four months after operation, with full muscle power and no sensory impairment.

\section{Conclusion}

Brachial plexus primary tumors as well as schwannomas of the head and neck are both uncommon entities, and combination of these conditions is quite rare. A majority of cases are presented as supraclavicular masses. However, if it arises from upper nerve roots of the brachial plexus, as our case, it may manifest as a mid-neck swelling that can be clinically indistinguishable from a lymph node. Close anatomical relations of the brachial plexus schwannoma with the critical structures in the cervical region, require an accurate preoperative planning to avoid catastrophic neurovascular complications. Despite the best precautions, a postoperative sensory or motor deficit commonly may occur. Although, fortunately, the vast majority of sensory injuries resolve spontaneously, motor injuries may require repair and nerve grafting, as in our case happened. 


\section{Consent}

Written informed consent was obtained from the patient for publication of this case report and any accompanying images. A copy of the written consent is available for review by the Editor-in-Chief of this journal.

\section{Competing Interest}

We declare that we have no financial or non-financial competing interests.

\section{Authors' Contributions}

AA was the primary surgeon, conceived of the report and obtained the patient's history and physical exam. HH assisted the surgery, reviewed the literature and drafted the manuscript. MC was surgeon's assistant and provided the images and their interpretations. FI reviewed the manuscript and contributed to its final form. All authors have been involved in writing this manuscript and have approved the final manuscript and its submission.

\section{References}

[1] Bradley, W. and Midah, R. (2008) Chap 49: Schwannoma. In: Midah, R., Ed., Surgery of Peripheral Nerve: A Case Based Approach, Thieme, New York, 231.

[2] Bocciolini, C., et al. (2005) Schwannoma of Cervical Sympathetic Chain: Assessment and Management. Acta Otorhinolaryngologica Italica, 25, 191-194.

[3] Jaafar, R., et al. (2012) Cervical Schwannoma: Report of Four Cases. Medical Journal of Malaysia, 67, 345-348.

[4] Patel, M.L., Sachan, R., Seth, G., et al. (2014) Schwannoma of the Brachial Plexus: A Rare Cause of Monoparesis. BMJ Case Reports.

[5] Soltani, A.M., et al. (2013) Neural Sheath Tumors of the Brachial Plexus: A Multidisciplinary Team-Based Approach. Annals of Plastic Surgery, 71, 80-83. http://dx.doi.org/10.1097/SAP.0b013e31827100d8

[6] Chiofalo, M.G., Longo, F., Marone, U., Franco, R., Petrillo, A. and Pezzullo, L. (2009) Cervical Vagal Schwannoma: A Case Report. Acta Otorhinolaryngologica Italica, 29, 33-35.

[7] Kumar, A. and Akhtar, S. (2011) Schwannoma of Brachial Plexus. Indian Journal of Surgery, 73, 80-81. http://dx.doi.org/10.1007/s12262-010-0141-1

[8] Rashid, M., et al. (2013) Schwannoma of the Brachial Plexus; Report of Two Cases Involving the C7 Root. Journal of Brachial Plexus and Peripheral Nerve Injury, 8, 12. http://dx.doi.org/10.1186/1749-7221-8-12

[9] Iaacconi, P., et al. (2012) Cervical Sympathetic Chain Schwannoma: A Case Report. Acta Otorhinolaryngologica Italica, 32, 133-136.

[10] Sérémé, M., Ouédraogo, A., Gyébré, Y. and Ouoba, K. (2014) Cervical Vagal Schwannoma: Difficulty of Diagnosis and Particularity of Treatment. The Internet Journal of Otorhinolaryngology, 16.

[11] Ahmadi-Yazdi, C. and Habashi, S. (2005) Schwannoma of Accessory Nerve: A Case Report. The Internet Journal of Otorhinolaryngology, 4.

[12] Chen, F., Miyahara, R., Matsunaga, Y. and Koyama, T. (2008) Schwannoma of the Brachial Plexus Presenting as an Enlarging Cystic Mass: Report of a Case. Annals of Thoracic and Cardiovascular Surgery, 14, 311-313.

[13] Somayaji, K.S.G., Rajeshwari, A. and Gangadhara, K.S. (2004) Schwannoma of the Brachial Plexus Presenting as a Cystic Swelling. Indian Journal of Otolaryngology and Head \& Neck Surgery, 56, 228-230.

[14] Haghari, S., İmerci, A., Koçak, M., Sürer, L. and Güney, E. (2013) A Case of Schwannoma Arising from Brachial Plexus in an Operated Patient with the Diagnosis of Cubital Tunnel Syndrome. Türkiye Fiziksel Tip ve Rehabilitasyon Dergisi, 59, 165-166. http://dx.doi.org/10.4274/tftr.10693

[15] Binder, D.K., Smith, J.S. and Barbaro, N.M. (2004) Primary Brachial Plexus Tumors: Imaging, Surgical, and Pathological Findings in 25 Patients. Neurosurgical Focus, 16, E11.

[16] Lusk, M.D., Kline, D.G. and Garcia, C.A. (1987) Tumors of the Brachial Plexus. Neurosurgery, 21, 439-453. http://dx.doi.org/10.1227/00006123-198710000-00001 